\title{
Downregulation of NKD1 in human osteosarcoma and its clinical significance
}

\author{
$\mathrm{XIANG} \mathrm{CHEN}^{1,2}$, PING XU ${ }^{2}$, JIANWEI ZHU ${ }^{1}$ and FAN LIU ${ }^{1}$ \\ ${ }^{1}$ Department of Orthopedics, Affiliated Hospital of Nantong University, Nantong, Jiangsu 226001; ${ }^{2}$ Department of Orthopedics, \\ Dongtai City Hospital of Traditional Chinese Medicine, Yancheng, Jiangsu 224200, P.R. China
}

Received January 29, 2016; Accepted February 21, 2017

DOI: $10.3892 / \mathrm{mmr} .2017 .7968$

\begin{abstract}
Naked cuticle homolog 1 (NKD1), a negative modulator of the canonical $\mathrm{Wnt} / \beta$-catenin pathway, is expressed in multiple normal tissues. However, there is little information regarding NKD1 expression in osteosarcoma. The aim of the present study was to explore the expression and clinicopathological significance of NKD1 in human osteosarcoma. In the present study, NKD1 protein and mRNA expression levels were detected by western blotting and reverse transcription-quantitative polymerase chain reaction, respectively. The results revealed that NKD1 expression levels were significantly lower in osteosarcoma tissues compared with normal bone tissue, and were significantly lower in patients with lung metastasis compared with patients without lung metastasis. In addition, with increasing Enneking stage, the NKD1 expression levels decreased. These data indicated that reduction of NKD1 may be associated with carcinogenesis, lung metastasis and Enneking stage in osteosarcoma. This interpretation is consistent with the results obtained from experiments on MG63 osteosarcoma cells in vitro. In order to explore the function of NKD1 in osteosarcoma, the expression of NKD1 in the human osteosarcoma MG-63 cell line was upregulated by transfection with an adenovirus containing an NKD1 vector. The results revealed that upregulation of NKD1 expression reduced the proliferation and migration of osteosarcoma cells by inhibiting expression of $\beta$-catenin, cyclin D1 and MMP-9 protein. These data suggested that the downregulation of NKD1 may be involved in the proliferation and migration of osteosarcoma cells through the activation of the canonical Wnt signaling pathway, and it may be a potential prognostic marker and therapeutic target for patients with osteosarcoma.
\end{abstract}

\section{Introduction}

Osteosarcoma is a histological form of primary bone cancer that primarily affects children, adolescents and young adults (1). At

Correspondence to: Dr Jianwei Zhu, Department of Orthopedics, Affiliated Hospital of Nantong University, 20 Xishi Road, Nantong, Jiangsu 226001, P.R. China

E-mail: zhujianwei_nt@163.com

Key words: osteosarcoma, NKD1, Wnt signaling pathway the point of diagnosis, in $\sim 20 \%$ of patients osteosarcoma has spread to the lungs and, in certain cases, to other bones $(2,3)$. The prognosis for patients with metastatic osteosarcoma remains poor despite the development of adjuvant and neoadjuvant chemotherapy (4). Current therapeutic strategies focus on the primary tumor and have limited efficacy for metastatic osteosarcoma (5). Treating metastatic osteosarcoma remains a challenge in oncology. Therefore, an improved understanding of the pathogenesis and biology of osteosarcoma may provide a rational basis for improving treatment effects, in particular for metastases (6).

Naked cuticle homolog 1 (NKD1) belongs to the naked cuticle (NKD) family (7). This family includes two members, NKD1 and NKD2. NKD2 is located on chromosome $5 \mathrm{p} 15.3$, which is frequently lost in human cancers (8). NKD2 was predicted to encode a 451-amino acid polypeptide. Zhao et al (9) revealed that NKD2, a negative regulator of Wnt signaling, suppressed tumor growth and metastasis in osteosarcoma. NKD1, located on chromosome 16q12.1, is widely expressed in multiple normal tissues (7). NKD1 was predicted to encode a 470-amino acid polypeptide, which serves as a negative regulator of the canonical $\mathrm{Wnt} / \beta$-catenin pathway by interacting with Dishevelled through its conservative EF-hand-like motifs (8). The EF-hand motif of NKD1 is required for its inhibitory function. NKD1 has previously been demonstrated to function as a passive antagonist of Wnt signaling, acting only when the Wnt signaling levels exceed a certain threshold $(9,10)$. Therefore, NKD1 may reduce aberrant Wnt signaling. However, in the absence of excess Wnt signaling, NKD1 activity is less apparent. Although increasing numbers of studies focus on NKD1, the involvement of NKD1 in cancer progression remains to be further addressed. Previous studies have demonstrated that NKD1 may be involved in embryonic development and tumorigenesis (11). Guo et al (12) demonstrated that specific NKD1 mutations may promote Wnt-dependent tumorigenesis. NKD1 is also frequently loss in human cancers, including breast cancer, lung cancer and colorectal cancer $(7,8,12,13)$. Lv et al $(7)$ demonstrated that reduction of NKD1 was significantly correlated with lymph node metastasis, histological grade and ER expression in breast cancer. Downregulation of NKD1 was associated with a lower cumulative postoperative 5 year survival rate (7). It has previously been suggested that down-regulation of NKD1 is associated with poor prognosis and increased invasive ability 
of non-small-cell lung cancer $(13,14)$. These data suggested that the downregulation of NKD1 may be involved in tumorigenesis and the progression of osteosarcoma.

However, the expression patterns and clinicopathological significance of NKD1 in patients with osteosarcoma remain unknown. The aim of the present study was to evaluate the expression of NKD1 in osteosarcoma tissues and cells by reverse transcription-quantitative polymerase chain reaction (RT-qPCR) and western blotting analysis, and to investigate whether reduced expression of NKD1 is involved in osteosarcoma development or progression. These data may provide scientific information useful for prognosis prediction and targeted therapies for osteosarcoma.

\section{Materials and methods}

Specimens. Fresh osteosarcoma tissues were collected from 21 patients who underwent surgical resection operations in the Affiliated Hospital of Nantong University (Nantong, China) between January 2009 and June 2015. At the same time, adjacent normal bone tissues were also collected. All these tissue specimens were immediately frozen in liquid nitrogen following surgery for subsequent experiments. The present study was approved by the Ethics Committee of the Affiliated Hospital of Nantong University (Nantong, China). Written, informed consent was obtained from all patients.

Reagents. All cell culture reagents were purchased from Gibco (Thermo Fisher Scientific, Inc., Waltham, MA, USA). Osteosarcoma cell line MG63 was obtained from the American Type Culture Collection (Manassas, VA, USA). Protein extraction and quantification kits, propidium iodide, dimethyl sulfoxide, Cell Counting Kit-8 and RNase A were from Beyotime Institute of Biotechnology (Haimen, China). The enhanced chemiluminescence kit was purchased from Pierce (Thermo Fisher Scientific, Inc.). Matrigel was purchased from Collaborative Biomedical Products, Inc. (Bedford, MA, USA). NKD1 (NCBI gene ID: 16303259) forward, 5'-atggagagagtgagcgaacc-3' and reverse, 5'-atcttgctggatgttgggga-3'; glyceraldehyde 3-phosphate dehydrogenase (NCBI gene ID: 182976) forward, 5'-aggtcg gagtcaacggattt-3' and reverse, 5'-atctcgctcctggaagatgg-3') were synthesized by Shanghai Generay Biotech Co., Ltd. (Shanghai, China). Adenovirus containing an NKD1 vector was purchased from Guangzhou RiboBio Co., Ltd. (Guangzhou, China). The rabbit NKD1 polyclonal antibody (cat. no. LS-C82447-50) was purchased from LifeSpan Biosciences, Inc. (Seattle, WA, USA). The mouse anti-human cyclin D1 (cat. no. 554180) monoclonal antibody was from BD Biosciences (San Jose, CA, USA). Rabbit anti-matrix metalloproteinase 9 (MMP9; cat. no. ABIN1873732) polyclonal antibody was purchased from Abnova (Taipei, Taiwan). Rabbit $\beta$-catenin (cat. no. ab23512) polyclonal antibody was purchased from Abcam (Cambridge, UK). Rabbit $\beta$-actin (cat. no. 253613) polyclonal antibodies were purchased from Abbiotec LLC (San Diego, CA, USA). Horseradish peroxidase-conjugated goat anti-rabbit (cat. no. 31460) and goat anti-mouse (cat. no. 31430) immunoglobulin G (IgG) polyclonal antibodies were from Invitrogen (Thermo Fisher Scientific, Inc.).

Cell culture and transfection. Osteosarcoma cell lines (MG63) were maintained in RPMI-1640 medium supplemented with
$10 \%$ fetal bovine serum (FBS) and $1 \%$ penicillin/streptomycin at $37^{\circ} \mathrm{C}$ in $5 \% \mathrm{CO}_{2}$. Human osteoblast cell lines (hFOB1.19) were cultured in Dulbecco's modified Eagle's medium: Nutrient mixture F-12 containing 10\% FBS and geneticin $(400 \mu \mathrm{g} / \mathrm{ml})$ at $34^{\circ} \mathrm{C}$ in a humidified $5 \% \mathrm{CO}_{2}$ incubator.

To enforce NKD1 expression in MG63 cells, MG63 cells were transfected with adenovirus containing an NKD1 vector, and served as an overexpression group. MG63 cells transfected with an adenovirus vector without NKD1 or without any treatment served as a control group and a blank group, respectively. The recommended confluency for MG63 cells at the day of transfection is $70-90 \%$. When this was reached, the complete medium was changed for serum-free RPMI-1640 medium. MG63 cells were transfected with adenovirus vector using Lipofectamine 2000 reagent (Thermo Fisher Scientific, Inc.) according to the manufacturer's protocol. The MG63 cells were harvested $48 \mathrm{~h}$ following transfection to measure the mRNA and protein levels.

Reverse transcription-quantitative polymerase chain reaction $(R T-q P C R)$. Total RNA was extracted from osteosarcoma tissues and cells using TRIzol reagent (Thermo Fisher Scientific, Inc.), according to the manufacturer's protocol. RNA concentrations were measured with an Ultrospec-3000 spectrophotometer (Milton Roy, Ivyland, PA, USA). The cDNA was synthesized from $1 \mu \mathrm{g}$ RNA using an iScript cDNA Synthesis kit (Bio-Rad Laboratories, Inc., Hercules, CA, USA), according to the manufacturer's protocols, and stored at $-20^{\circ} \mathrm{C}$ until further use. The qPCR reactions were performed using the iTaq Fast SYBR-Green Supermix (Bio-Rad Laboratories, Inc.) according to the manufacturer's protocol. Quantitative mRNA expression data were acquired and analyzed using an Applied Biosystems 7500 real-time PCR system (Applied Biosystems, Thermo Fisher Scientific, Inc.) with the PCR cycling conditions as follows: An initial step at $95^{\circ} \mathrm{C}$ for $5 \mathrm{~min}$ for enzyme activation, followed by 40 cycles of $3 \mathrm{sec}$ at $95^{\circ} \mathrm{C}, 30 \mathrm{sec}$ at $60^{\circ} \mathrm{C}$ and finally at $72^{\circ} \mathrm{C}$ for $10 \mathrm{~min}$. The cycle threshold $(\mathrm{Cq})$ values, corresponding to the PCR cycle number at which fluorescence emission reached a threshold above baseline emission, were determined and the relative mRNA expression was calculated using the $2^{-\Delta \Delta \mathrm{Cq}}$ method (15). All the experiments were repeated in triplicate.

Western blotting. Total proteins were extracted from osteosarcoma tissues and cells using RIPA buffer [150 $\mathrm{mM} \mathrm{NaCl}$, $1 \%$ NP40, 0.5\% sodium deoxycholate, $0.1 \%$ SDS, $50 \mathrm{mM}$ Tris (pH 7.9), $10 \mathrm{mM} \mathrm{NaF}$, phenylmethylsulfonyl fluoride and $1 \mathrm{X}$ protease inhibitors (complete cocktail tablets; Roche Diagnostics, Basel, Switzerland)] and quantified using a BCA assay kit (Beyotime Institute of Biotechnology). A total of $40 \mu \mathrm{g}$ proteins were loaded per lane and underwent $12 \%$ SDS-PAGE. Then, the proteins were transferred to a polyvinylidene difluoride (PVDF) membrane. The PVDF membrane was blocked in 5\% skimmed milk in TBS for $1 \mathrm{~h}$ at room temperature and then incubated with primary antibodies against the following antigens: NKD1, cyclin D1, $\beta$-catenin, MMP9 and $\beta$-actin, diluted in 5\% skimmed milk in TBST (0.1\% Tween-20), all 1:2,000, at $4^{\circ} \mathrm{C}$ overnight. Subsequently, the PVDF membrane was washed in TBST $(0.1 \%$ Tween- 20$)$ and probed with corresponding secondary antibodies (goat 
anti-rabbit $\operatorname{IgG}$ and goat anti-mouse $\operatorname{IgG}$ ) diluted (both $1: 2,000)$ in $5 \%$ skimmed milk in TBST $(0.1 \%$ Tween-20) for $2 \mathrm{~h}$ at room temperature. Following washing with TBST $(0.1 \%$ Tween-20), band intensity was determined using an ECL kit. The relative expression of target protein was evaluated with the gray value ratio of target protein content to $\beta$-actin content (target protein $/ \beta$-actin) by densitometry analysis software Quantity-one (version 4.62; Bio-Rad Laboratories, Inc.). All the experiments were repeated in triplicate.

Cell viability and cell cycle analysis. Water soluble tetrazolium salt (WST)-8 is more useful than MTT for analyzing cell viability, as it is reduced to soluble formazan in the presence of electronic coupling reagent by dehydrogenase in the mitochondria. The more cells proliferate, the darker the color is. The more toxic the cell is, the lighter the color is. Cell proliferation was determined using WST-8 dye according to manufacturer's protocol pf the CCK- 8 kit. Briefly, MG- 63 cells were seeded at a density of 3,000 cells per well in 96 well microplates. $10 \mu \mathrm{l}$ CCK-8 solution and $100 \mu \mathrm{l}$ RPMI-1640 medium were added to each well, and the plates were incubated for $2 \mathrm{~h}$ at $30^{\circ} \mathrm{C}$. The optical density was detected at a wavelength of $450 \mathrm{~nm}$ by a microplate reader. All the experiments were repeated in triplicate.

MG-63 cells were cultured in serum-free medium for $24 \mathrm{~h}$ to synchronize and then cultured in complete RPMI-1640 medium for $24 \mathrm{~h}$. The MG-63 cells were then harvested and fixed in $0.5 \mathrm{ml} 70 \%$ precooled ethanol, and kept at $4^{\circ} \mathrm{C}$ until staining. The fixed MG-63 cells were washed and then pretreated with RNAse $(10 \mu \mathrm{g} / \mathrm{ml})$ for $30 \mathrm{~min}$ at $37^{\circ} \mathrm{C}$. Then, $10 \mu \mathrm{l}$ propidium iodine was added to the cell suspension at a final concentration of $100 \mu \mathrm{g} / \mathrm{ml}$, and incubation was continued for $30 \mathrm{~min}$ at room temperature in the dark prior to analysis. The cell cycle was detected by flow cytometry (FACSCalibur; BD Biosciences, Franklin Lakes, NJ, USA) and CellQuest software (version 6.0; BD Biosciences) was used to analyze the results. For cell cycle analysis, data were expressed as fractions of cells in different cycle phases, and the experiment was repeated 3 times.

Matrigel invasion assay. Cell invasion was examined by Matrigel invasion assay. Briefly, $200 \mu 1$ RPMI-1640 medium containing $1 \times 10^{3}$ MG63 cells was added to the upper part of each Transwell chamber. The bottom of the Transwell chamber was filled with $500 \mu 1$ RPMI 1640 medium containing 10\% FBS, which acted as chemoattractant. MG63 cells were incubated at $37^{\circ} \mathrm{C}$ in a humidified $5 \% \mathrm{CO}_{2}$ and $95 \%$ air incubator for $48 \mathrm{~h}$. Following $48 \mathrm{~h}$ incubation, the MG63 cells on the top surface of the chamber were removed using a cotton swab. Migrated cells were fixed with $4 \%$ paraformaldehyde for $15 \mathrm{~min}$ at room temperature and stained with $0.05 \%(\mathrm{w} / \mathrm{v})$ crystal violet solution for $10 \mathrm{~min}$, followed by observation under a Leica DMI3000 B inverted light microscope (Leica Microsystems GmbH, Wetzlar, Germany) and got the average number by counts from 6 random fields of view per filter The experiments were performed in triplicate and the number of invaded cells were calculated.

Statistical analysis. SPSS 17.0 software (SPSS, Inc., Chicago, IL, USA) was used to analyze the data in the present study.
The $\chi^{2}$ test and one-way analysis of variance followed by Bonferroni's test were used for statistical analysis in the present study. $\mathrm{P}<0.05$ was considered to indicate a statistically significant difference.

\section{Results}

Downregulation of NKD1 in osteosarcoma. RT-qPCR was used to examine the expression of NKD1 mRNA in osteosarcoma. The results revealed that the NKD1 mRNA expression rate in osteosarcoma tissues was $27.78 \%$, which was significantly lower than that in normal bone tissues (88.89\%; P<0.05; Table I). The NKD1 mRNA expression rate decreased gradually as Enneking stage increased (16) $(\mathrm{P}<0.05$; Table I). At the same time, the NKD1 mRNA expression rate from patients with positive lung metastasis were significantly lower compared with patients with negative lung metastasis $(\mathrm{P}<0.05$; Table I). However, the NKD1 mRNA expression rate in osteosarcoma was independent of the patient's sex, age, or tumor size $(\mathrm{P}>0.05$; Table I). The western blotting results were similar to the RT-qPCR results, which revealed that NKD1 protein expression was downregulation in osteosarcoma tissues compared with normal bone tissues $(\mathrm{P}<0.05$; Fig. 1), decreased gradually with the increase of Enneking stages $(\mathrm{P}<0.05$; Fig. 1), and was reduced in the carcinoma tissues from patients with positive lung metastasis compared with patients with negative lung metastasis $(\mathrm{P}<0.05$; Fig. 1). These data implied that the downregulation of NKD1 may be involved in the tumorigenesis and progression of osteosarcoma.

NKD1 inhibits osteosarcoma MG63 cell viability. In order to explore the effect of NKD1 on the characteristics of osteosarcoma cells, NKD1 expression in MG63 cells was enforced by transfection with adenovirus containing an NKD1 vector. The results from RT-qPCR and western blotting demonstrated that NKD1 mRNA and protein was significantly upregulated in the overexpression group compared with the control group and blank group $(\mathrm{P}<0.05$; Fig. 2). These data indicated that NKD1 expression was effectively enforced in osteosarcoma MG63 cells.

MG63 cell viability was determined using WST-8 dye. The results indicated that MG63 cell viability in the overexpression group was significantly decreased compared with the control and blank groups ( $\mathrm{P}<0.05$; Fig. 2). Thus, MG63 cell viability was significantly inhibited when NKD1 was upregulated by transfection with adenovirus containing an NKD1 vector. The opposite inference also stands: NKD1 downregulation may be associated with increased MG63 cell viability.

NKD1 inhibits osteosarcoma MG63 cell cycle progression. The results from the flow cytometry assay demonstrated that the percentage of MG63 cells at $\mathrm{G}_{0} / \mathrm{G}_{1}$ phase in the overexpression group was increased compared with the control group and blank group ( $\mathrm{P}<0.05$; Fig. 3), and the percentage of MG63 cells at the $\mathrm{S}$ and $\mathrm{G}_{2} \mathrm{M}$ phases in the overexpression group was decreased compared with the control group and blank group $(\mathrm{P}<0.05$; Fig. 3 ). Based on the above results, it was possible to conclude that NKD1 downregulation may be associated with progression of the MG63 cell cycle. 
Table I. NKD1 mRNA expression in osteosarcoma tissues and its association with clinicopathological characteristics.

\begin{tabular}{|c|c|c|c|c|}
\hline Clinicopathological characteristic & Cases & NKD1 mRNA positive rate $(\%)$ & $\chi^{2}$ & P-value \\
\hline Normal tissue & 18 & $16(88.89)$ & 13.8286 & 0.000 \\
\hline Carcinoma tissue & 18 & $5(27.78)$ & & \\
\hline \multicolumn{5}{|l|}{ Sex } \\
\hline Male & 11 & $2(18.18)$ & 1.2983 & 0.255 \\
\hline Female & 7 & $3(42.86)$ & & \\
\hline \multicolumn{5}{|l|}{ Age (years) } \\
\hline$<20$ & 10 & $3(30.00)$ & 0.0554 & 0.814 \\
\hline$\geq 20$ & 8 & $2(25.00)$ & & \\
\hline \multicolumn{5}{|l|}{ Size of primary carcinoma $(\mathrm{cm})$} \\
\hline$<8$ & 12 & $3(25.00)$ & 0.1385 & 0.710 \\
\hline$\geq 8$ & 6 & $2(33.33)$ & & \\
\hline \multicolumn{5}{|l|}{ Enneking stage } \\
\hline I stage & 5 & $4(80.00)$ & 9.7398 & 0.008 \\
\hline II stage & 7 & $1(14.29)$ & & \\
\hline III stage & 6 & $0(00.00)$ & & \\
\hline \multicolumn{5}{|l|}{ Lung metastasis } \\
\hline Negative & 11 & $5(45.45)$ & 4.4056 & 0.036 \\
\hline Positive & 7 & $0(00.00)$ & & \\
\hline
\end{tabular}

NKD1, naked cuticle homolog 1 .

NKD1 suppresses osteosarcoma MG63 cell invasion. Matrigel invasion assays were used to test the invasive ability of MG63 cells. The results revealed that the overexpression group had less MG63 cells invading into the Matrigel membrane compared with the control group and blank group ( $\mathrm{P}<0.05$; Fig. 4$)$. The number of invaded MG63 cells did not differ significantly between the control group and blank group ( $P>0.05$; Fig. 4). These data indicated that NKD1 may suppress osteosarcoma MG63 cell invasion.

NKD1 suppresses the expression of $\beta$-catenin, cyclin D1 and MMP9 in MG63 cells. The present study demonstrated that the downregulation of NKD1 may be involved in the tumorigenesis and progression of osteosarcoma, and NKD1 overexpression may inhibit osteosarcoma MG63 cell viability, proliferation and invasion. In the present study, western blotting analysis indicated that $\beta$-catenin was downregulated in the overexpression group compared with the control group and blank group $(\mathrm{P}<0.05$; Fig. 5), which indicated that NKD1 suppressed the expression of $\beta$-catenin. $\beta$-catenin is a positive regulator of the canonical Wnt signaling pathway, therefore, it was inferred that NKD1 inhibited the proliferation and invasion of osteosarcoma cell through activating the canonical Wnt pathway. To test this hypothesis, the expression levels of cyclin D1 and MMP9, target genes of the canonical Wnt pathway, were determined. The results revealed that the expression of cyclin D1 and MMP9 was significantly inhibited in the overexpression group compared with the control group and blank group $(\mathrm{P}<0.05$; Fig. 5), which indicated that NKD1 suppressed the proliferation and invasion of osteosarcoma cells through inhibition of the canonical Wnt signaling pathway.

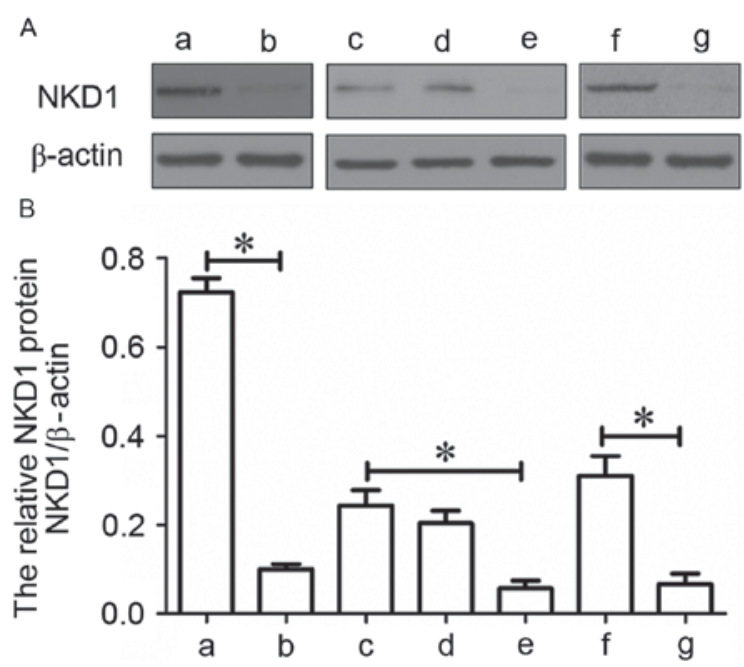

Figure 1. NKD1 protein expression levels. (A) NKD1 protein expression in osteosarcoma tissues as analysed by western blot, with (B) quantification. ${ }^{*} \mathrm{P}<0.05$, with comparisons indicated by lines. NKD1, naked cuticle homolog 1 ; a, normal bone tissue; b, osteosarcoma tissue; c, Enneking stage I; d, Enneking stage II; e, Enneking stage III; f, lung metastasis negative; g, lung metastasis positive.

\section{Discussion}

NKD1 is an antagonist of the canonical Wnt/ $\beta$-catenin pathway (8). Dysregulation of Wnt signaling is at the root of multiple diseases, including cancer (17). Guo et al (12) demonstrated that specific NKD1 mutations may promote Wnt-dependent tumorigenesis. NKD1 is widely expressed in multiple normal tissues. However, it is frequently lost in human 


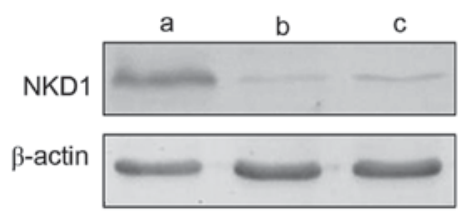

B

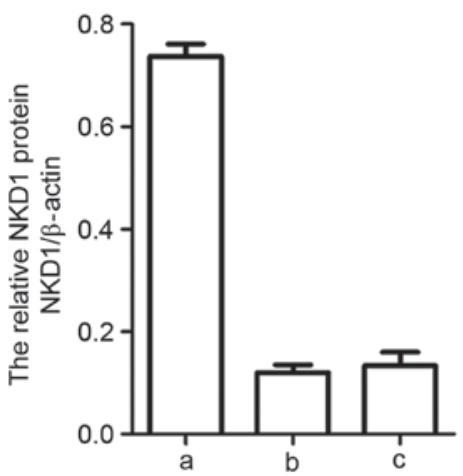

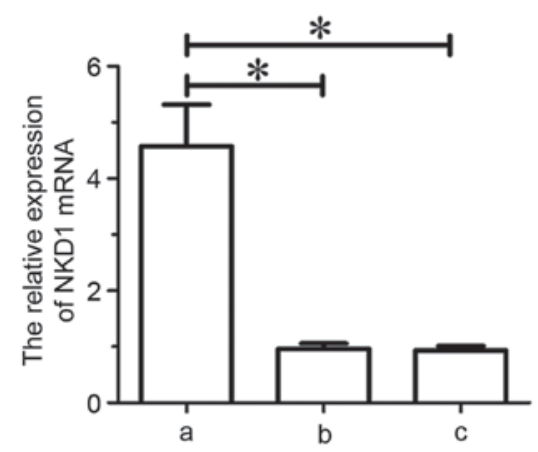

D

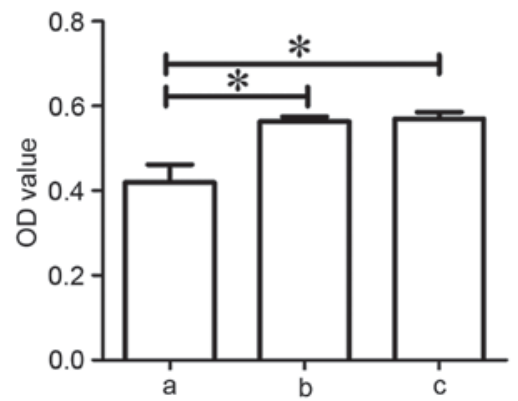

Figure 2. The expression of NKD1 in MG63 cells and the effect of NKD1 on MG63 cell viability. (A) NKD1 protein expression in MG63 cells, with (B) quantification. (C) NKD1 mRNA expression levels in MG63 cells. (D) NKD1 inhibits MG63 cell viability, as determined by water soluble tetrazolium salt-8 staining. ${ }^{*} \mathrm{P}<0.05$, with comparisons indicated by lines. NKD1, naked cuticle homolog 1; a, NKD1 overexpression group; b, control group; c, blank group.

A
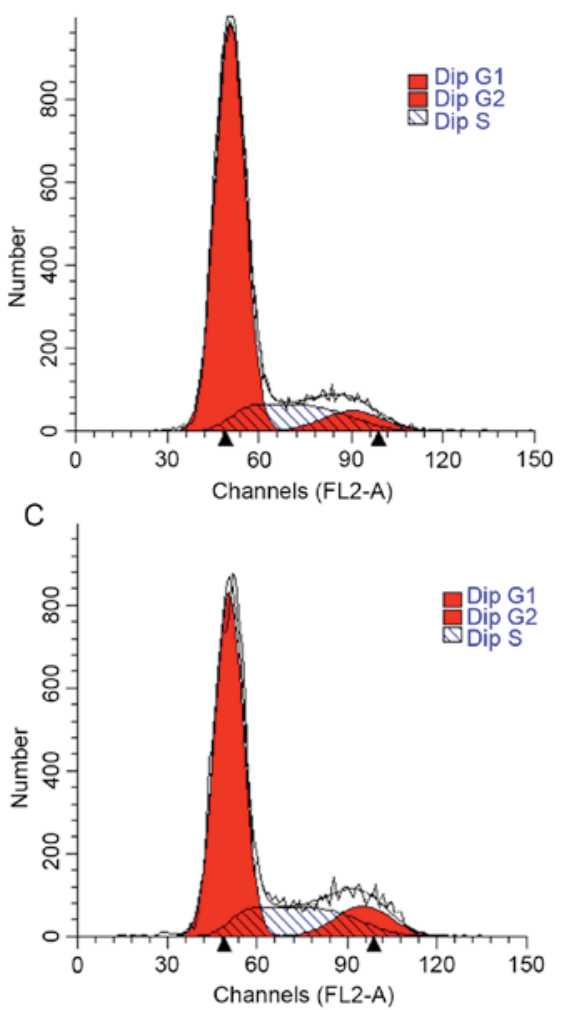

B

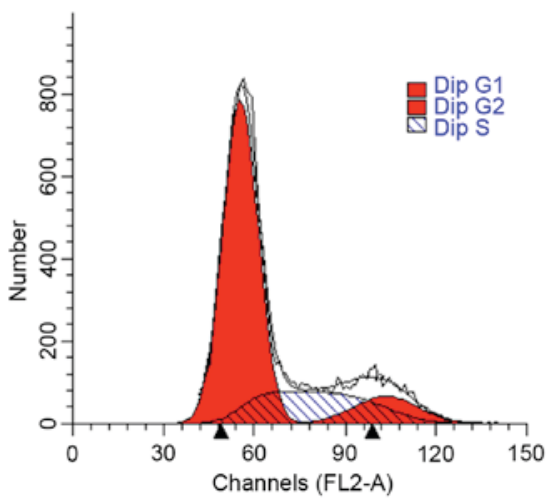

D

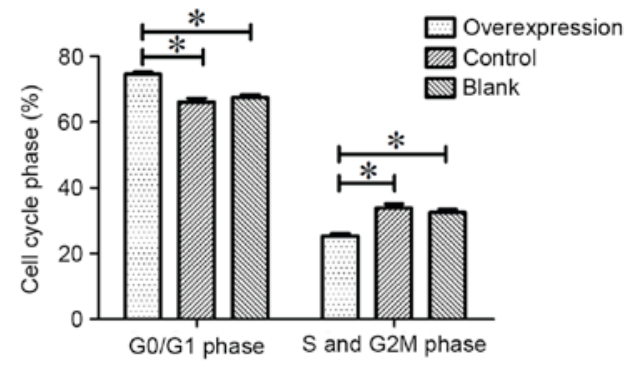

Figure 3. NKD1 suppresses MG63 cell proliferation. Representative cell cycle distribution graphs for (A) NKD1 overexpression group, (B) control group and (C) blank group. (D) Cell cycle analysis. "P<0.05, with comparisons indicated by lines. All experiments were repeated three times with three replicates each. NKD1, naked cuticle homolog 1 .

cancers, including breast cancer, gastric cancer, lung cancer and colorectal cancer $(7,8,12,13)$. Although there are increasing numbers of reports concerning NKD1, the expression pattern and clinicopathological significance of NKD1 in patients with osteosarcoma remains unclear, and the involvement of NKD1 in cancer progression still needs to be further addressed. 

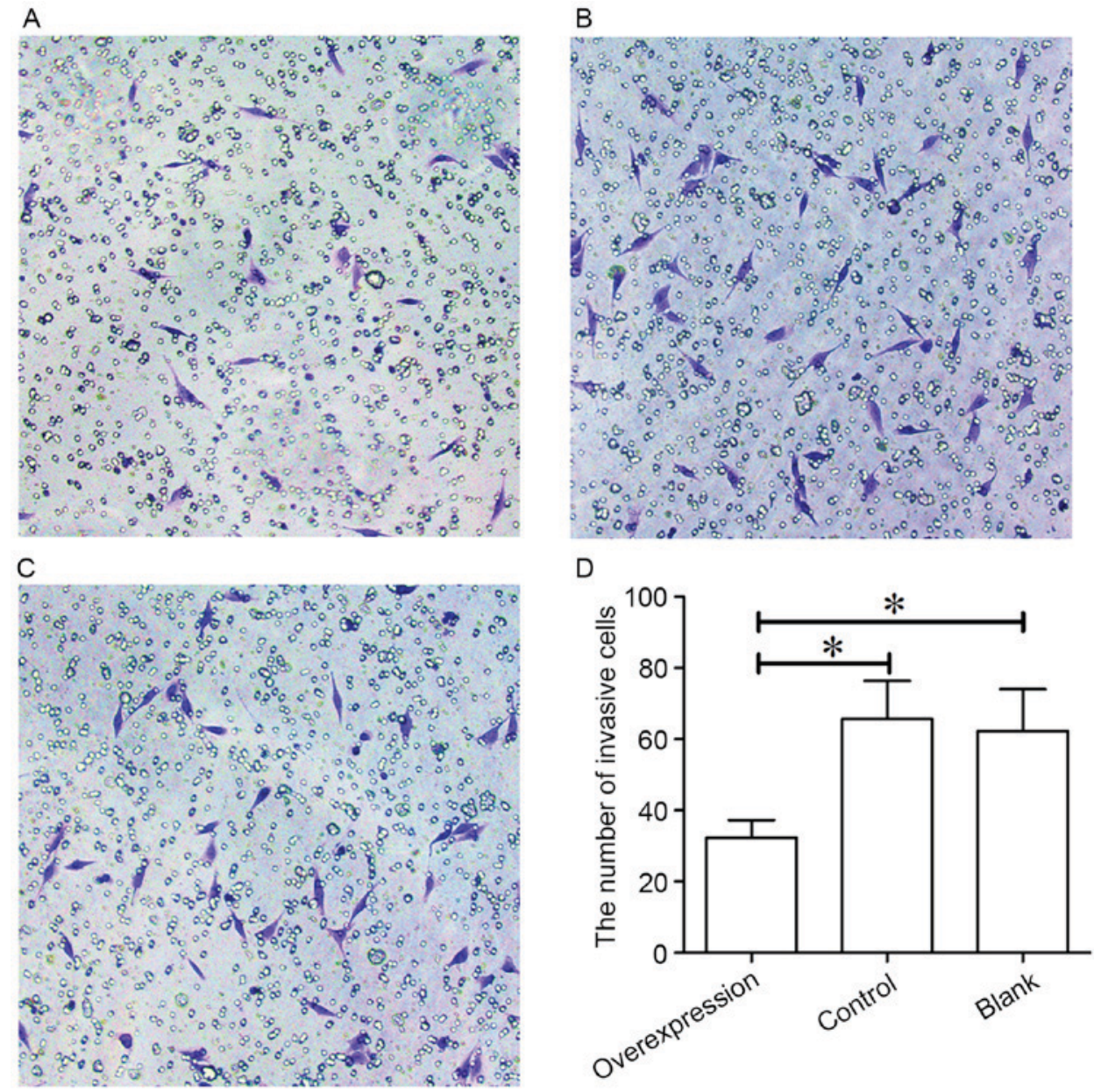

Figure 4. NKD1 inhibits MG63 cell invasion. Representative cell invasion images of (A) NKD1 overexpression group, (B) control group and (C) blank group with (D) quantification. Magnification, $\mathrm{x} 40 .{ }^{*} \mathrm{P}<0.05$, with comparisons indicated by lines. All experiments were repeated three times with three replicates each. NKD1, naked cuticle homolog 1.

A

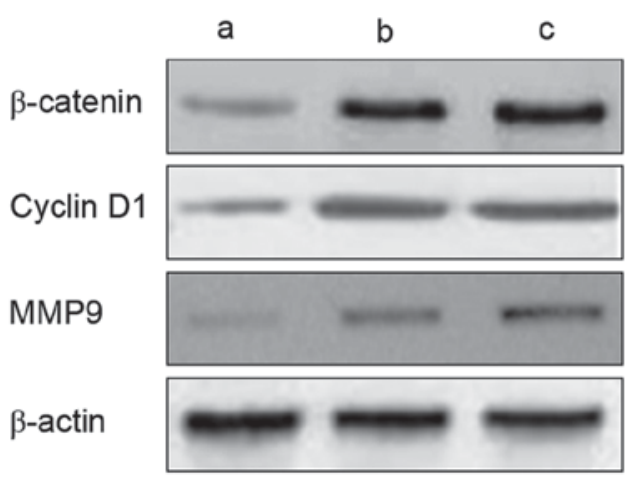

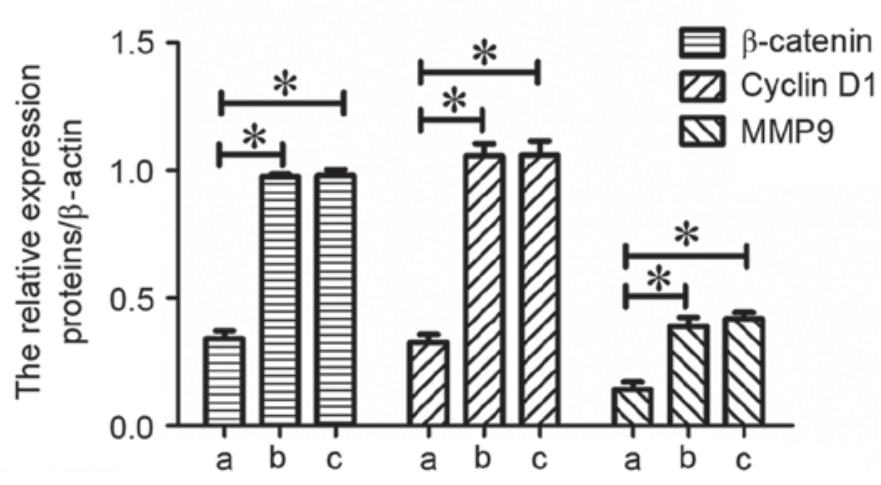

Figure 5. NKD1 suppresses the expression of $\beta$-catenin, cyclin D1 and MMP9 in MG63 cells. (A) Representative western blotting images with (B) quantification. "P<0.05, with comparisons indicated by lines. All experiments were repeated three times with three replicates each. NKD1, naked cuticle homolog 1; a, NKD1 overexpression group; b, control group; $c$, blank group; MMP9, matrix metalloproteinase 9.

In the present study, NKD1 expression was demonstrated to be downregulated in osteosarcoma tissues, and to decrease as Enneking stages increased. The data revealed that the expression profile of NKD1 in osteosarcoma was similar to the expression profile of NKD1 in breast cancer, gastric cancer, lung cancer and colorectal cancer $(7,8,12,13)$. These data indicated that the downregulation of NKD1 may be involved in the tumorigenesis and progression of osteosarcoma. Activated Wnt/ $\beta$-catenin signaling promotes cellular proliferation, and Wnt inhibition reduces proliferation $(18,19)$. Mutant NKD1 is unable to inhibit Wnt signaling, and mutant NKD1 may stabilize $\beta$-catenin and promote cell proliferation (12). The results of the present study demonstrated that MG63 cell viability was significantly inhibited when NKD1 was upregulated. NKD1 downregulation may also 
be associated with increased MG63 cell viability. The percentage of MG63 cells at the G0/G1 phase in the overexpression group was higher, and the percentage of MG63 cells at the S and G2 M phase in the overexpression group was lower than in the control group and blank group, which implied that NKD1 downregulation may be associated with the progression of MG63 cell cycle.

Previous studies have demonstrated that reduction of NKD1 is significantly correlated with lymph node metastasis, increased invasive ability and poor prognosis of multiple types of cancer $(7,13,14)$. Activation of $W n t / \beta$-catenin signaling promotes the invasion of several types of cancer cells $(20,21)$. Suppression of Wnt/ $\beta$-catenin signaling inhibits cancer cells invasion $(22,23)$. Lv et al (7) demonstrated that NKD1 may be involved in invasion in human breast cancer. The results of the present study demonstrated that expression of NKD1 was reduced in the carcinoma tissues from patients with positive lung metastasis compared with patients with negative lung metastasis, which suggested that reduction of NKD1 was associated with metastasis of osteosarcoma. The in vitro data indicated that NKD1 may suppress osteosarcoma MG63 cell invasion, which implied that NKD1 downregulation may enhance the invasion of osteosarcoma MG63 cell. The data obtained by the present study were in keeping with the results obtained by Zhang et al (14), whose study demonstrated that NKD1 knockdown increased the invasion of lung cancer cells.

NKD1 has previously been reported to be an antagonist of the canonical Wnt/ $\beta$-catenin pathway (24). The results of the present study also demonstrated that $\beta$-catenin was downregulated in the NKD1 overexpression group, which indicated that NKD1 inhibited the expression of $\beta$-catenin. $\beta$-catenin is a positive regulator of the canonical Wnt signaling pathway, therefore, it was inferred that NKD1 inhibited the proliferation and invasion of osteosarcoma cells through activating the canonical Wnt signaling pathway. To test this hypothesis, the target genes of the canonical Wnt pathway, cyclin D1 and MMP9, were analyzed. The expression of cyclin D1 and MMP9 was significantly inhibited in the NKD1 overexpression group, which indicated that NKD1 suppressed the expression of cyclin D1 and MMP9 in osteosarcoma cells. These results verified the hypothesis. These data suggested that the downregulation of NKD1 may be involved in the proliferation and migration of osteosarcoma cells through the activation of the canonical Wnt signaling pathway and may be a potential prognostic marker and therapeutic target for patients with osteosarcoma.

\section{Acknowledgements}

The present study was supported by the Natural Science Foundation of Jiangsu Province (grant no. BK20131199), the 55th Batch of China Postdoctoral Science Foundation (grant no. 2014M551640), Nantong Science and Technology Innovation Program (grant no. MS22016008) and the Project of Jiangsu Provincial Health and Family Planning Commission (grant no. H201524).

\section{References}

1. Sampson VB, Yoo S, Kumar A, Vetter NS and Kolb EA: MicroRNAs and potential targets in osteosarcoma: Review. Front Pediatr 3: 69, 2015.
2. Endo-Munoz L, Evdokiou A and Saunders NA: The role of osteoclasts and tumour-associated macrophages in osteosarcoma metastasis. Biochim Biophys Acta 1826: 434-442, 2012.

3. Lamora A, Talbot J, Bougras G, Amiaud J, Leduc M, Chesneau J, Taurelle J, Stresing V, Le Deley MC, Heymann MF, et al: Overexpression of smad7 blocks primary tumor growth and lung metastasis development in osteosarcoma. Clin Cancer Res 20: 5097-5112, 2014.

4. Kimura H, Tsuchiya H, Shirai T, Nishida H, Hayashi K, Takeuchi A, Ohnari I and Tomita K: Caffeine-potentiated chemotherapy for metastatic osteosarcoma. J Orthop Sci 14: 556-565, 2009.

5. He JP, Hao Y, Wang XL, Yang XJ, Shao JF, Guo FJ and Feng JX: Review of the molecular pathogenesis of osteosarcoma. Asian Pac J Cancer Prev 15: 5967-5976, 2014.

6. Poletajew S, Fus L and Wasiutyński A: Current concepts on pathogenesis and biology of metastatic osteosarcoma tumors. Ortop Traumatol Rehabil 13: 537-545, 2011 (In English, Polish).

7. Lv ZD, Zhang L, Liu XP, Jin LY, Dong Q, Li FN, Wang HB and Kong B: NKD1 down-regulation is associated with poor prognosis in breast invasive ductal carcinoma. Int J Clin Exp Pathol 8: 4015-4021, 2015.

8. Jia Y, Cao B, Yang Y, Linghu E, Zhan Q, Lu Y, Yu Y, Herman JG and Guo M: Silencing NKD2 by promoter region hypermethylation promotes gastric cancer invasion and metastasis by up-regulating SOX18 in human gastric cancer. Oncotarget 6: 33470-33485, 2015.

9. Zhao S, Kurenbekova L, Gao Y, Roos A, Creighton CJ, Rao P, Hicks J, Man TK, Lau C, Brown AM, et al: NKD2, a negative regulator of Wnt signaling, suppresses tumor growth and metastasis in osteosarcoma. Oncogene 34: 5069-5079, 2015.

10. Angonin D and Van Raay TJ: Nkd1 functions as a passive antagonist of Wnt signaling. PLoS One 8: e74666, 2013.

11. Li Q, Ishikawa TO, Miyoshi H, Oshima M and Taketo MM: A targeted mutation of Nkd1 impairs mouse spermatogenesis. J Biol Chem 280: 2831-2839, 2005.

12. Guo J, Cagatay T, Zhou G, Chan CC, Blythe S, Suyama K, Zheng L, Pan K, Qian C, Hamelin R, et al: Mutations in the human naked cuticle homolog NKD1 found in colorectal cancer alter Wnt/Dvl/beta-catenin signaling. PLoS One 4: e7982, 2009.

13. Ahn S, Hwangbo W, Kim H and Kim CH: Naked cuticle Drosophila 1 expression in histologic subtypes of small adenocarcinoma of the lung. Korean J Pathol 47: 211-218, 2013.

14. Zhang S, Wang Y, Dai SD and Wang EH: Down-regulation of NKD1 increases the invasive potential of non-small-cell lung cancer and correlates with a poor prognosis. BMC Cancer 11: 186, 2011.

15. Livak KJ and Schmittgen TD: Analysis of relative gene expression data using real-time quantitative PCR and the 2(-Delta Delta C(T)) method. Methods 25: 402-408, 2001.

16. He L, Yang Z, Zhou J and Wang W: The clinical pathological significance of FRAT1 and ROR2 expression in cartilage tumors. Clin Transl Oncol 17: 438-445, 2015.

17. Larraguibel J, Weiss AR, Pasula DJ, Dhaliwal RS, Kondra R and Van Raay TJ: Wnt ligand-dependent activation of the negative feedback regulator Nkd1. Mol Biol Cell 26: 2375-2384, 2015.

18. Cheng AS, Lau SS, Chen Y, Kondo Y, Li MS, Feng H, Ching AK, Cheung KF, Wong HK, Tong JH, et al: EZH2-mediated concordant repression of Wnt antagonists promotes $\beta$-catenin-dependent hepatocarcinogenesis. Cancer Res 71: 4028-4039, 2011.

19. Stewart DJ: Wnt signaling pathway in non-small cell lung cancer. J Natl Cancer Inst 106: djt356, 2014

20. Cai J, Feng D, Hu L, Chen H, Yang G, Cai Q, Gao C and Wei D: FAT4 functions as a tumour suppressor in gastric cancer by modulating Wnt $/ \beta$-catenin signalling. Br J Cancer 113 : $1720-1729,2015$

21. Ye GD, Sun GB, Jiao P, Chen C, Liu QF, Huang XL, Zhang R, Cai WY, Li SN, Wu JF, et al: OVOL2, an inhibitor of WNT signaling, reduces invasive activities of human and mouse cancer cells and is downregulated in human colorectal tumors. Gastroenterology 150: 659-671.e16, 2016.

22. Fan Y and Guo Y: Knockdown of eIF3D inhibits breast cancer cell proliferation and invasion through suppressing the Wnt $/ \beta$-catenin signaling pathway. Int J Clin Exp Pathol 8: 10420-10427, 2015.

23. Deng Z, Wang L, Hou H, Zhou J and Li X: Epigenetic regulation of IQGAP2 promotes ovarian cancer progression via activating Wnt/ß-catenin signaling. Int J Oncol 48: 153-160, 2016.

24. Zhang S, Li J, Yin ZY, Liu PG, Zhao WX, Xie CR, Zhao BX and Wang XM: Expression pattern and clinicopathologic significance of NKD1 in human primary hepatocellular carcinoma. APMIS 123: 315-320, 2015 